\title{
A review on the reduction of data taken from a microlensing event
}

\author{
S Sajadian, B Adami, and M R Mohammadi
}

Department of Physics, Isfahan University of Technology, Isfahan, Iran

\author{
E-mail: s.sajadian@cc.iut.ac.ir
}

(Received 03 April 2019 ; in final form 24 September 2019)

\begin{abstract}
Data reduction is one the most important process of researches conducted in astronomy and astrophysics. Data reduction process includes all steps which convert the crude astronomical images to the astrophysical events. These steps are similar for different astronomical events in which the brightness of one star changes with time, although they may be different in some details. This paper is concerned with learning or even doing data reduction of each astronomical event. Data reduction of microlensing events contains 7 stages in which the crude images will be first calibrated. Then, some of the best calibrated images are chosen for making the reference images. The reference image helps to compare all taken images to indicate how the source light changes versus time. For this propose all images should be registered to the reference images. Then, all images should be taken to the same scale. Finally, the resulted images are subtracted from the reference image to find the light curve of that desired source star.
\end{abstract}

Keywords: reduction of astronomical images, gravitational microlensing event

For full article, refer to the Persian section 